\section{Predictive markers for response to interferon $\beta$ therapy in MS}

Up to $50 \%$ of patients with multiple sclerosis (MS) relapse despite receiving treatment with interferon $\beta$ (IFN- $\beta$ ). Two recent studies have investigated pharmacological and genetic markers that could predict the patients who will respond optimally to IFN- $\beta$ therapy and, thus, potentially identify those who would benefit from alternative treatment regimens.

In the first study, Malucchi et al. clinically validated three biological markers commonly used to detect response to IFN- $\beta$ therapy: binding antibodies (BAbs), which are thought to alter receptor-mediated functions of IFN- $\beta$; neutralizing antibodies (NAbs), which could potentially decrease the treatment efficacy of IFN- $\beta$; and myxovirus-resistance protein A (MxA, or MX1), a protein that acts as a measure of IFN- $\beta$ bioactivity. This retrospective study analyzed 137 patients with definite MS who had been receiving IFN- $\beta$ therapy for $\geq 3$ years. Patients had undergone blood tests at baseline to determine that they were initially negative for BAbs and NAbs and had levels of $M \times A(M X 1)$ gene expression similar to those of controls, and again after $12 \pm 3$ months of IFN- $\beta$ therapy in order to assign, on the basis of predefined thresholds, positive or negative status for each of $M x A$ mRNA, BAb and NAb.

Time to first relapse was significantly shorter in $M x A$-negative patients than in $M x A$-positive individuals (median 7 months vs $>3$ years; $P<0.0001$ ) and was also shorter in NAb-positive patients than in those who were NAb-negative (median 8 months vs $>3$ years; $P=0.0013$ ), indicating that the clinical efficacy of IFN- $\beta$ was reduced in $M x A$-negative and NAb-positive patients. There was no significant difference between the numbers of BAb-positive and BAb-negative patients who were relapse free.

In the second study, Byun et al. identified and validated genes in which allelic variation might influence response to IFN- $\beta$ therapy. Singlenucleotide polymorphism (SNP) microarrays with genome-wide coverage were used to assess population allele frequencies in pooled DNA samples from 206 patients with clinically definite relapsing-remitting MS treated with IFN- $\beta$ therapy who had been prospectively followed up for at least 2 years; 99 were responsive and 107 were nonresponsive to IFN- $\beta$ therapy. Significance-based ranking identified 35 top candidate SNPs that appeared at different frequencies in patients who had responded to IFN- $\beta$ therapy and those who had not.

When individual patient genotypes were compared in the second stage of the analysis, differences were found between responders and nonresponders in 29 of the 35 candidate SNPs. When genotype data from an additional 81 patients were added to this analysis, expression of 18 of the 35 candidate SNPs remained significantly different between the groups. Many of the candidate SNPs identified in the joint analysis were located in genes associated with ion channels and signal transduction pathways as well as with extracellular matrix proteins; the others were located in intergenic regions.

The results from the Malucchi et al. study indicate that changes in $M x A$ expression and (to a lesser degree) in serum NAb levels after 1 year of IFN- $\beta$ therapy might predict relapse in patients with MS. Byun and co-workers suggest that genotyping for the specific gene sets identified in their study could likewise predict response to IFN- $\beta$ therapy.

Original articles Malucchi S et al. (2008) Predictive markers for response to interferon therapy in patients with multiple sclerosis. Neurology 70: 1119-1127

Byun E et al. (2008) Genome-wide pharmacogenomic analysis of the response to interferon beta therapy in multiple sclerosis. Arch Neurol 65: 337-344

\section{Listening to music can help patients in early stroke recovery}

Results from animal studies have suggested that an enriched auditory environment can enhance recovery from neural damage, but data regarding this effect in humans are limited. Särkämö et al. assessed the long-term effects of listening to music on the cognitive and emotional functions of patients who were recovering from a left or right hemisphere middle cerebral artery stroke.

The single-blind, randomized, controlled study recruited 60 patients from a hospital in Helsinki, Finland, from March 2004 to May 2006. The patients were randomized to a music group, a language group, or a control group ( $n=20$ in each). Patients in the first two groups were instructed to listen to, respectively, music recordings or audio books 